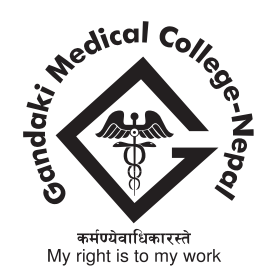

Journal of

GANDAKI MEDICAL COLLEGENEPAL (J-GMC-N)

J-GMC-N | Volume $\mathbf{1 0}$ | Issue $\mathbf{0 1}$ January-June $\mathbf{2 0 1 7}$

\section{Fungal Infections (Mycoses): Dermatophytoses (Tinea, Ringworm)}

\author{
Reddy KR \\ Professor \& Head \\ Microbiology Department \\ Gandaki Medical College \& Teaching Hospital, Pokhara, Nepal
}

Medical Mycology, a study of fungal epidemiology, ecology, pathogenesis, diagnosis, prevention and treatment in human beings, is a newly recognized discipline of biomedical sciences, advancing rapidly. Earlier, the fungi were believed to be mere contaminants, commensals or nonpathogenic agents but now these are commonly recognized as medically relevant organisms causing potentially fatal diseases.

The discipline of medical mycology attained recognition as an independent medical speciality in the world sciences in 1910 when French dermatologist Raymond Jacques Adrien Sabouraud (1864 - 1936) published his seminal treatise Les Teignes. This monumental work was a comprehensive account of most of then known dermatophytes, which is still being referred by the mycologists. Thus he has laid down the foundation of the field of Medical Mycology. He has been aptly referred as the "Father of Medical Mycology".

There are significant developments in treatment modalities of fungal infections and we have achieved new prospects. However, till 1950s there was no specific antifungal agent available. Nystatin was discovered in 1951 and subsequently amphotericin B was introduced in 1957 and was sanctioned for treatment of human beings. In the 1970s, the field was dominated by the azole derivatives. Now this is the most active field of interest, where potential drugs are being developed to treat fungal infections. By the end of the $20^{\text {th }}$ century, the fungi have been reported to be developing drug resistance, especially among yeasts.

Medical mycology has now completed one and half century (mid $19^{\text {th }}$ and $20^{\text {th }}$ ) and entered into $21^{\text {st }}$ century. There have been lots of developments during this period, particularly after the advent of modern molecular techniques. But still many issues remain unresolved before the mycologists. Dermatophytoses continues to be a major public health problem. The human pathogenic fungi are now recognized to cause morbidity and mortality among humans and also animals. They have emerged as important etiologic agents of opportunistic infections as well as full-fledged diseases as true pathogens.

There is a steady increase in the number of patients suffering from life-threatening fungal infections, and there is an emergence of newer fungal pathogens and drug resistance due to:

- Prolonged and indiscriminate use of antibiotic therapy

- Immunosuppressive corticosteroid therapy 
- $\quad$ Aggressive use of anticancer drugs

- Bone marrow and organ transplantation

- Immunodeficiency (AIDS)

- Metabolic disorders (E.g., Diabetes mellitus)

Based on the wide spectrum of adaptability, various fungi causing human mycoses can be categorized into pathogenic fungi, opportunistic fungi, toxigenic fungi and allergenic fungi. According to the site of primary involvement, the fungal diseases of man can be classified into five types (Table 1).

Table 1: Various fungal diseases and their causative fungi

\begin{tabular}{|c|c|c|c|}
\hline & Infection category & Fungal disease & Causative fungi \\
\hline \multirow[t]{5}{*}{1} & \multirow{5}{*}{$\begin{array}{l}\text { Superficial } \\
\text { mycoses }\end{array}$} & Tinea nigra & Hortaea werneckii \\
\hline & & Black piedra & Piedrai hortae \\
\hline & & Dermatomycoses & $\begin{array}{l}\text { Scytalidium dimidiatum, } \\
\text { Dermatophytes }\end{array}$ \\
\hline & & Onychomycosis & $\begin{array}{l}\text { Scytalidium dimidiatum, } \\
\text { Dermatophytes }\end{array}$ \\
\hline & & Keratomycosis & $\begin{array}{l}\text { Curvularia sp, Alternaria sp, } \\
\text { Bipolaris sp, Fonsecaea pedrosoi, } \\
\text { Aureobasidium pullulans }\end{array}$ \\
\hline \multirow[t]{5}{*}{2} & \multirow[t]{5}{*}{$\begin{array}{l}\text { Subcutaneous } \\
\text { mycoses }\end{array}$} & Eumycetoma & $\begin{array}{l}\text { Exophiala jeanselmei, } \\
\text { Curvularia geniculata }\end{array}$ \\
\hline & & Chromoblastomycosis & $\begin{array}{l}\text { Fonsecaea pedrosoi, } \\
\text { Fonsecaea compacta, Philalophora } \\
\text { verrucosa, Cladophialophora } \\
\text { carrionii, Rhinocladiella aquaspersa }\end{array}$ \\
\hline & & Rhinosporidiosis & Rhinosporidium seeberi \\
\hline & & Phaeohyphomycosis & $\begin{array}{l}\text { Wangiella dermatitidis, } \\
\text { Exophiala jeanselmei, } \\
\text { Cladophialophora bantiana, } \\
\text { Chaetomium sp }\end{array}$ \\
\hline & & Sporotrichosis & Sporotrichum schenkii \\
\hline \multirow[t]{4}{*}{3} & \multirow[t]{4}{*}{ Systemic mycoses } & Histoplasmosis & Histoplasma capsulatum \\
\hline & & Coccidioidomycosis & Coccidioides immitis \\
\hline & & Paracoccidioidomycosis & Paracoccidioides brasiliensis \\
\hline & & Blastomycosis & Blastomyces dermatitidis \\
\hline \multirow[t]{6}{*}{4} & \multirow[t]{6}{*}{$\begin{array}{l}\text { Opportunistic } \\
\text { mycoses }\end{array}$} & Mucormycosis & $\begin{array}{l}\text { Mucor sp, Rhizopus sp, } \\
\text { Fusarium sp. }\end{array}$ \\
\hline & & Pneumocystosis & Pneumocystis jirovecii (P. carinii) \\
\hline & & Candidiasis & Candida albicans \\
\hline & & Cryptococcosis & Cryptococcus neoformans \\
\hline & & Penicilliosis & Penicillium marneffei \\
\hline & & Aspergillosis & Aspergillus sp. \\
\hline 5 & $\begin{array}{l}\text { Miscellaneous } \\
\text { mycoses }\end{array}$ & Allergic rhinosinusitis & $\begin{array}{l}\text { Alternaria alternata, } \\
\text { Bipolaris hawaiiensis, } \\
\text { Curvularia lunata, } \\
\text { Exserohilum rostratum }\end{array}$ \\
\hline
\end{tabular}




\section{SUPERFICIAL MYCOSES}

The superficial cutaneous fungal infections involve the outer most layers of skin and its appendages like hair and nails. These are among the most commonly encountered infections and prevalent in most parts of the world. The causative agents colonize cornified layer of epidermis or supra-follicular proteins of hair and do not penetrate into deeper anatomical sites. There is little tissue damage by the causative fungi and the immune response is also of at low threshold. The patients invariably neglect such types of infections and seek medical attention for cosmetic reasons. The dermatophytes are by far the most significant fungi because of their widespread involvement of population at large and their prevalence all over the world.

\section{CUTANEOUS MYCOSES}

Dermatophytoses are the most common types of cutaneous fungal infections seen in humans and animals affecting skin, hair and nails. These are caused by a group of filamentous fungi, closely related to each other antigenically, taxonomically, morphologically and physiologically; with a capacity to invade keratinized tissues of skin and its appendages and are collectively known as dermatophytes. Other frequently used terms like tinea and ringworm infections are synonyms of dermatophytoses. The infections caused by nondermatophytic fungi involving the skin are called as dermatomycoses whereas involving hair and nail are called piedra and onychomycosis respectively.

The dermatophytes are assigned to three main genera namely Trichophyton, Microsporum and Epidermophyton. All these three fungi belong to division Deuteromycetes, class Hyphomycetes, order Moniliales and family Moniliaceae. The three genera are differentiated by their conidial morphology.

1. Genus Trichophyton: This genus includes 24 species, some of them are saprophytes. They produce microconidia abundantly that may be globose or pyriform and are borne singly along the sides of the hyphae or in grape like clusters. Macroconidia are rare, if present they are elongated, pencil shaped. They infect skin, hair and nails. Examples of human pathogenic species of this genus are T. rubrum, T. mentagrophytes, T. violaccum, T. tonsurans, T. schoenleinii, T. verrucosum, T. concentricum, T. equinum, T. simii, T. soudanense etc.

2. Genus Microsporum: This genus includes 17 species, some of which are saprophytes. Macroconidia are abundant that are spindle or fusiform shaped with rough walls with 3 - 10 septa. Microconidia are scanty and are usually arranged singly along the sides of hyphae. They infect skin and hair; nails are not usually affected. Human pathogenic species are M. audouinii, M. gypseum, M. canis, M. nanum, M. ferrugineum, M. equinum, M. gallinae etc.

3. Genus Epidermophyton: This genus includes only two species, out of which only one is pathogenic. Microconidia are absent. Macroconidia are abundant, borne in clusters with smooth, thick walls and two to seven septa. They are broadly clavate. They infect skin and hair but not nails. Human pathogenic species is E. floccosum. 
Fig 1: Conidial morphology of dermatophytes

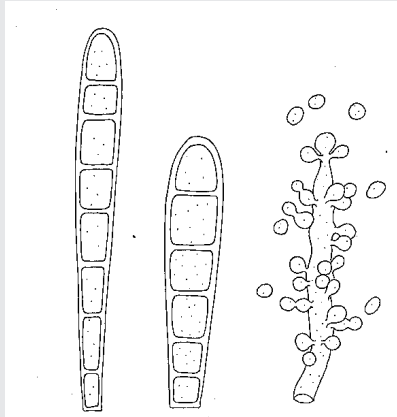

A

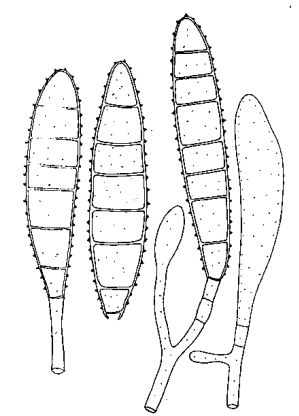

B

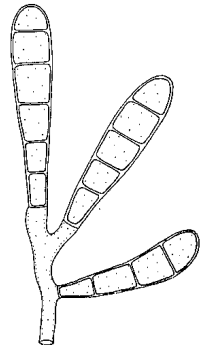

C

A: T. mentagrophytes B: M. gypseum C: E. floccosum

All these fungi reproduce asexually and for some sexual states are also reported. The terms anamorph (asexual) and teleomorph (sexual) is often used to describe the taxonomic status of the organism. The anamorphs, those lack a known sexual state have been placed in a separate major high-level taxon called Deuteromycetes or fungi imperfecti or mitosporic fungi.

\section{Epidemiology}

The prevalence of dermatophytoses is governed by environmental conditions, personal hygiene, and individual's susceptibility from place to place. The isolation of different species of dermatophytes also varies markedly from one ecological niche to another depending on their primary natural habitat. Ecologically, dermatophytes have been divided into geophilic, zoophilic and anthropophilic species.

1. Geophilic dermatophytes: The natural habitat of these species is the soil. Exposure to soil is the main source of infection for humans and animals. E.g. T. ajelloi, T. terrestre, M. gypseum, M. nanum, M. cookie, M. persicolor, M. fulvum.

2. Zoophilic dermatophytes: They inhabit domestic and wild animals such as birds, dogs, cats, horses, cattle etc. Human infections are usually acquired by direct contact with infected animals. Certain zoophilic species may be isolated more often from soil and from fur of apparently healthy animals. E.g. T. mentagrophytes, T. verrucosum, T. equinum, T. simii, M. canis, M. equinum, M. gallinae.

3. Anthropophilic dermatophytes: The fungal species exclusively affecting humans are known as anthropophilic. The migration of labor, troop movement, emigration and international travel played important roles in spreading of these fungi. E.g. T. rubrum, $T$. mentagrophytes, T. violaceum, T. schoenleinii, T. tonsurans, T. concentricum, T. soudanense, M. audouinii, M. ferrugineum, E. floccosum.

Many saprophytic soil fungi are closely related to dermatophytes, sharing the ability to utilize keratin as a growth substrate and it is believed that the dermatophytes have evolved from these keratinophilic soil fungi. During this evolutionary process various dermatophyte species have become adapted to a particular host and this has eventually led to the development of the epidemiological groups of anthropophilic, zoophilic and geophilic species. As dermatophytosis is prevalent throughout the world, it primarily depends on the 
habits and living conditions of the people as infection is transmitted through fomites. This is the only true contagious fungal infection in real sense. The arthrocondia are parasitic propagules and survive in the environment for a pretty long time.

Some species of the dermatophytes are endemic in certain parts of the world and have a limited geographic distribution. Trichophyton soudanense, T. gourvilii and T. yaoundei are geographically restricted to Central and West Africa. Microsporum ferrugineum predominates in Japan and surrounding areas. T. concentricum is confined to islands in the South Pacific and a small area in Central and South America. However, the increasing mobility of the world's population is disrupting several of these epidemiological patterns. In recent times, T. tonsurans has replaced M. audouinii as the principal agent of tinea capitis in USA, may be because of mass migration of individuals from Mexico and other Latin American countries where T. tonsurans predominates. The most common etiologic agents of dermatophytoses in the Western countries are T. rubrum and M. canis. Microsporum distortum is a rare cause of tinea capitis in New Zealand and Australia and infection in animals such as dogs. It is similar to and possibly is a variant of M. canis.

Ring worm infections are very common among adults; may be attributed to increased physical activity and increased opportunity for exposure and hormonal pattern. Males are more commonly affected than females; may be due to increased outdoor physical activities as they are the ones who go out to earn for their families and increased opportunity for exposure to the fungi.

In general, dermatophytoses are found to be common among people of low socioeconomic status and in rural areas. This may be due to poor hygienic conditions i.e., common practice of sharing clothes and bathing towels of other ring worm patients without washing them properly and also due to poor nutritional value.

The disease occurrence was observed to be common in summer and minimum in winter, indicating that sweating in summer may be a predisposing factor for dermatophytoses.

The prevalence of dermatophytoses varies in India. In 1900, Dr. Powell reported the first case of dermatophytosis from Assam, India. In India the commonest species isolated are T. rubrum followed by T. mentagrophytes and E. floccosum. T. violaceum is the commonest species causing tinea capitis. Tinea corporis and tinea cruris are the commonest varieties seen in India. Tinea corporis is commonly seen around the waistline of Indian men and women who wear dhoti and sarees. Similarly tinea pedis is seen among those who wear shoes for long hours like athletes.

\section{Clinical features}

Infections caused by dermatophytes are clinically classified, on the basis of location of the lesions on the body. Although different body sites may be affected, each focus of infection is generally a result of local inoculation. The invading dermatophyte grows in a centrifugal manner, forming irregular rings with inflammatory borders with some clearing in central area of lesion. The term tinea (Latin: worm or moth) describes the annular (ring like) lesions that resemble a worm burrowing at the margin. The clinical manifestations are classified as follows.

i. Tinea capitis: Ringworm of scalp involves infection of hair and scalp and presents as following two clinical types (Fig 1).

i. Inflammatory: Keroin, favus 
ii. Non-inflammatory: Black dot, seborrheic dermatitis like

Favus is an acute inflammatory reaction of the hair follicle, which leads to the formation of dense crusts (scutula) around the hair follicles leading to alopecia and scarring. Some zoophilic dermatophytes induce a severe inflammatory and hypersensitivity reaction known as keroin. Hair invasion is of two types (Fig 2):

a. Ectothrix: The fungus infects the hair shaft at midfollicle and forms a sheath of hyphae and arthroconidia 2 - $3 \mu \mathrm{m}$ in diameter that surrounds the shaft. The infected hair become lustreless, brittle and hair filaments break off at the level of scalp to give an appearance of partial alopecia. Commonest species associated with ectothrix infection of hair are M. audouinii, M. canis, M. ferrugineum, M. gypseum.

b. Endothrix: The hyphae invade hair follicle, then the hair shaft, and form numerous arthroconidia within the hair shaft. The infected hair becomes greyish white, breaks off easily to give black-dot appearance. T. violaceum, T. tonsurans are the common species causing this infection.

Fig 1: Tinea capitis
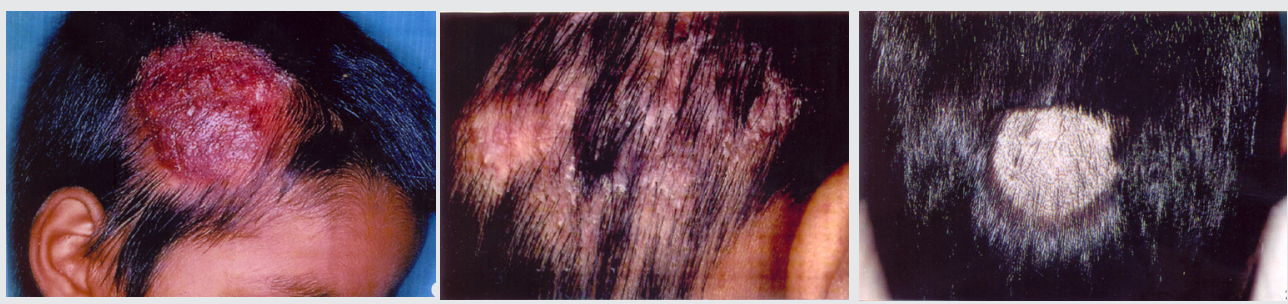

Fig 2: Ectothrix and endothrix infections
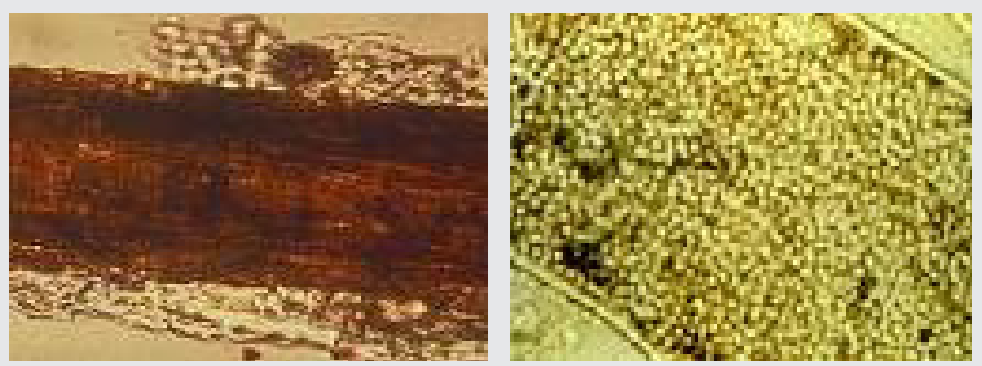

Tinea capitis is predominantly observed among children and young adults. This may be due abstinence from application of oil to hair containing fatty acids which has inhibiting effect on fungi. In addition, it may also be due to the presence of thymus or its remnants and lack of fungicidal and fungistatic secretions by adrenal glands during childhood. The resistance of adults to tinea capitis may also be due to increased secretory activity of the sebaceous glands at puberty and the antifungal activity of the $\mathrm{C}_{7}-\mathrm{C}_{11}$ saturated fatty acids in sebum (Undecylenic acid, an unsaturated $\mathrm{C}_{11}$ fatty acid widely used for topical therapy).

ii. Tinea corporis: Ringworm of glabrous skin. The lesions are well marginated with raised erythematous borders. The annular, scaly patches may coalesce to form large area of chronic infection (Fig 3). Commonest species causing this type of infection are $T$. rubrum, T. mentagrophytes, T. tonsurans.

Tinea corporis is observed to be predominant among people with previous family history of disease. The disease may be transmitted by direct contact with other infected individuals. Tinea capitis is the second most important clinical type seen among people with previous family history of disease. It is because these diseases may be transmitted through fomites such as comb, hair brushes, bedding, pillows, clothes, towels or 
furniture etc. In addition, tinea corporis can be attributed to poor personal hygiene and heavy manual work.

iii. Tinea cruris (Jock itch, Dhobie's itch): Ringworm of inguinal area involving the groin, perianal, perineal areas often involving the upper thigh (Fig 4). Common species involved are T. rubrum, T. mentagrophytes, E. floccosum.

Tinea cruris is mainly seen among students as they mostly wear synthetic tight under-garments in which sweat does not get absorbed and long standing moisture predisposes to fungal infection.

Fig 3: Tinea corporis
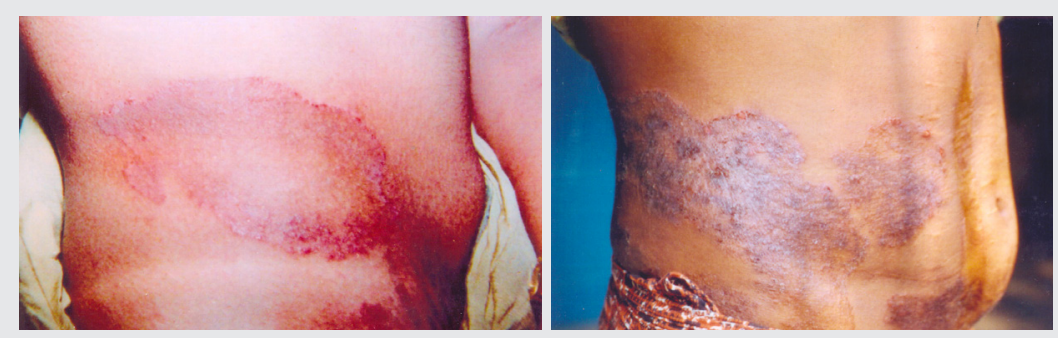

Fig 4: Tinea cruris

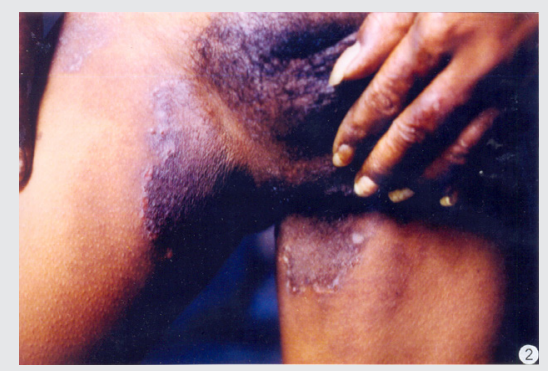

iv. Tinea pedis (Athlete's foot): Ringworm infection of feet involving interdigital webs and sole. The most common clinical manifestation is intertriginous form associated with maceration, scaling, fissuring and erythema which presents with itching and burning (Fig 5). Commonest infecting species are E. floccosum, T. rubrum, T. mentagrophytes.

Tinea pedis is common among athletes and office workers. It is due to constant wearing of shoes with synthetic nylon socks which does not absorb sweat.

v. Tinea manuum: Ringworm infection of palms and interdigital areas of hands and lesions present as diffuse hyperkeratotic areas (Fig 6). Commonest etiologic agent is $T$. rubrum.

Fig 5: Tinea pedis

Fig 6: Tinea manuum (Ringworm of palms)

(Ringworm of foot, Athlete's foot)
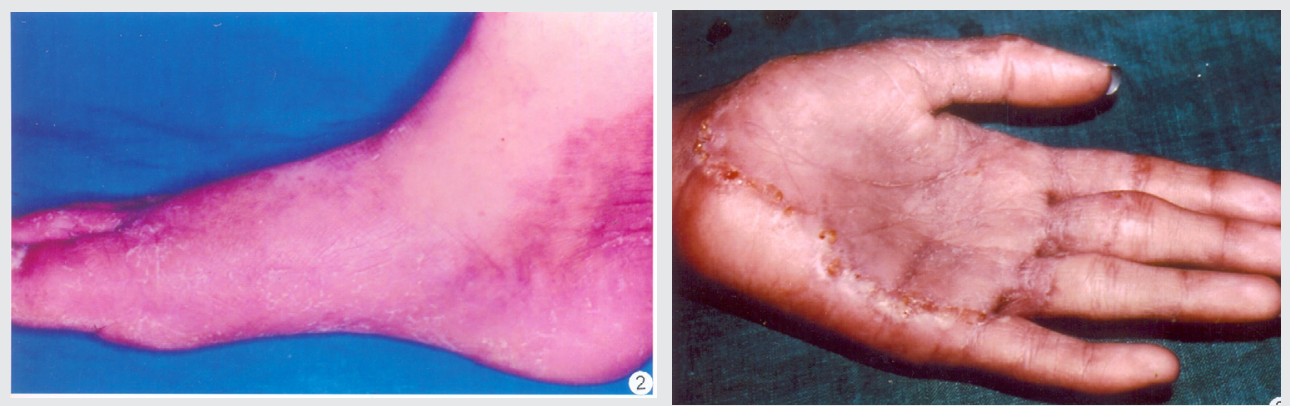

vi. Tinea barbae (Barber's itch): Ringworm infection of coarse hair of beard and moustache (Fig 7). The lesions are inflammatory and pustular. Commonest etiologic 
agents are T. verrucosum, T. mentagrophytes.

Tinea barbae is exclusively seen only in males, and the disease may be acquired from barber shops through contaminated instruments.

viii. Tinea faciei: Ringworm infection of glabrous skin of face, excluding beard area (Fig 8). Commonest species associated with this disease are T. rubrum, T. mentagrophytes, T. tonsurans.

Fig 7: Tinae barbae

(Ringworm of beard and moustache, Barber's itch)

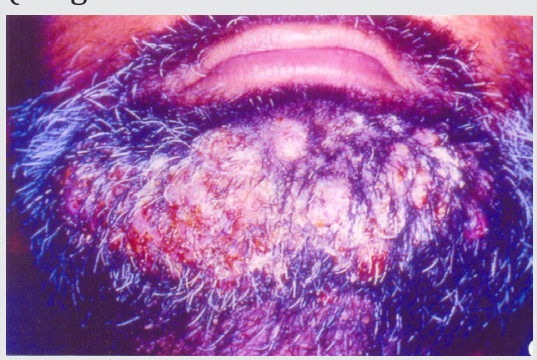

Fig 8: Tinea faciei (Ringworm of face)

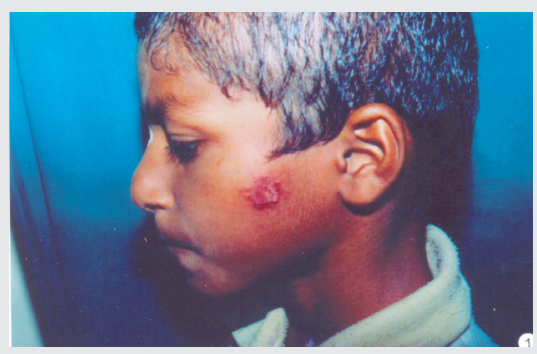

vii. Tinea unguium: Ringworm infection of nail plate. Distal subungual infection is the commonest pattern and involves nail bed and underside of nail in distal portion. The nail plate is brittle, friable, thickened and may crack because of piling up of subungual debris. The color of nail is often brown or black (Fig 9). Commonest species causing tinea unguium are T. rubrum, T. mentagrophytes, E. floccosum.

Tinea unguium is common among housewives and servant maids due to practice of cleaning the cowshed bare handed, washing the house hold utensils with ash and frequent dipping of hands in soap water; all of which enhance the chances of fungal infection.

Fig 9: Tinea unguium

(Ringworm of nails)
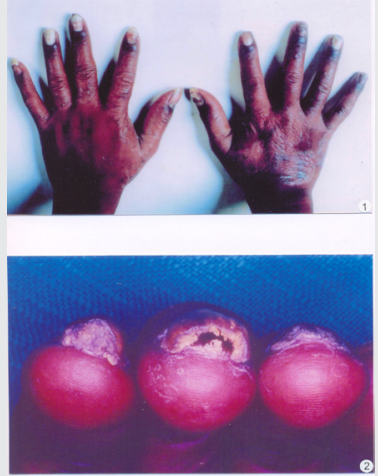

Fig 10: Tinea corporis and tinea unguium) (Mixed infection)

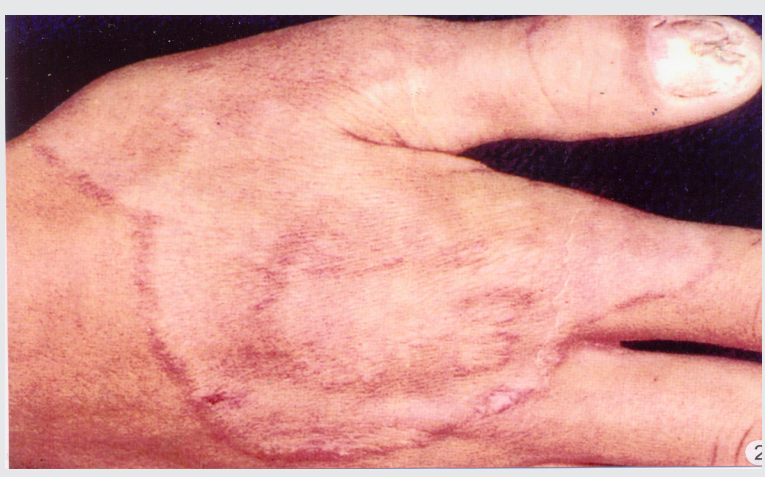

ix. Tinea imbricata: It is characterized by polycyclic, concentrically arranged rings of papulosquamous patches of scales scattered over and often covering most of the body. It is found in limited geographical areas (Pacific and Fizi islands) and caused by $T$. concentricum.

xi. Dermatophytid or ID reactions: The "id" reactions are allergic reactions to ring worm and occur in large variety of clinical forms. The most common location is that a patient with tinea pedis suddently develops itching and burning of both hands, usually beginning at the sides of fingers near the digital crease. In a few days, vesicles appear that enlarge to bullae and lesions spread to palm. The vesicles are sterile. The reaction may be the result of fungal products being absorbed from the skin and itching is the only symptom (Fig 11). 
Fig 11: Id reactions

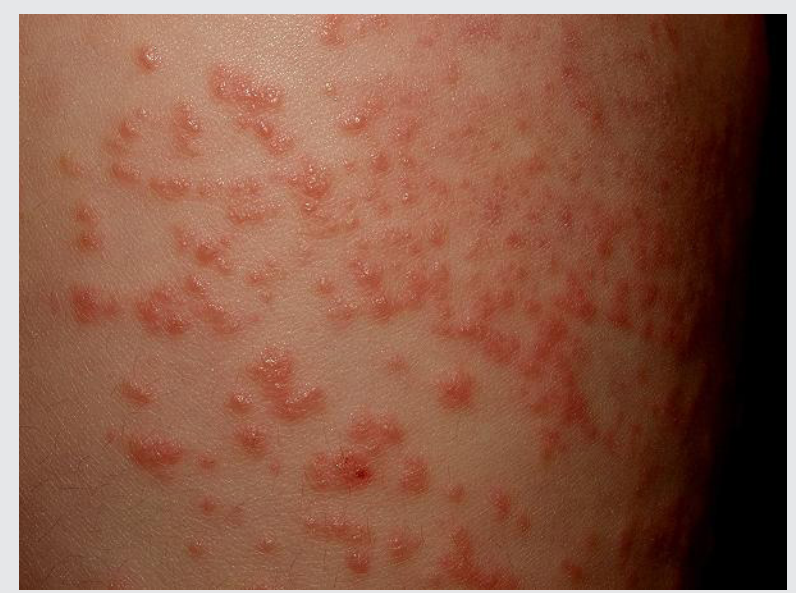

\section{Immunology}

The host response to a dermatophyte plays a major role in the pathogenesis of dermatophytosis. The immune system and skin work together to prevent and cure infections at skin surface by inhibiting fungal growth and accelerating epidermopoiesis so that fungal hyphae are removed from the host by normal replacement process of skin, hair and nail. The clinical manifestations are mostly due to the immune response of the host to the invading fungal species. Some degree of acquired resistance both humoral and cellular has been noted following dermatophyte infection. The appearance of an acute inflammatory reaction in ring worm correlates with development of cell mediated immunity (Type IV delayed hypersensitivity) to the dermatophyte, which can be elicited by an intradermal test with trichophytin antigen.

Several factors, which can account for natural resistance (nonspecific defense) to dermatophytoses in humans and animals are known. Natural resistance to dermatophytes in humans clearly appears after puberty. The natural resistance is due to the presence of fungistatic long chain fatty acids in the sebum at puberty. Internal organs of humans and animals are naturally resistant to dermatophytoses. A fungistatic substance called serum factor of normal individuals and animals is believed to limit the growth of dermatophytes to the stratum corneum. The serum factor is an unstable, dialyzable, heat labile component of fresh serum and tissue fluid.

\section{Laboratory diagnosis}

Several standardized methods are available for the clinical diagnosis and laboratory diagnosis of dermatophytoses. The following specimens are collected by a sterile scalpel blade depending on clinical type. Infected hair is selected by exposure to Wood's lamp (UV light). Infected hair will fluoresce under Wood's lamp. Therefore, Wood's lamp is used for the diagnosis of tinea capitis. It is an UV lamp emitting radiation at $365 \mathrm{~nm}$ (Fig 12).

- $\quad$ Skin scrapings from edges of ringworm lesions

- $\quad$ Nail clippings

- hair stubs 
Fig 12: Wood's lamp

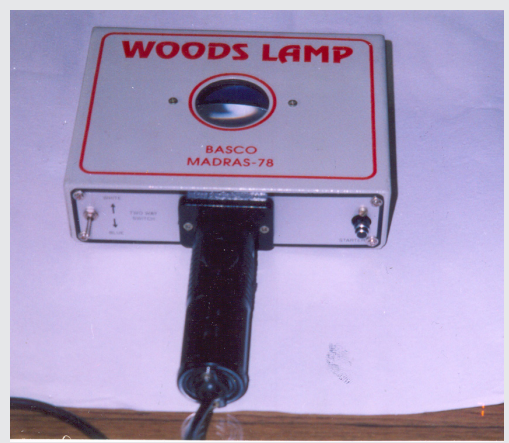

The most important laboratory methods are direct microscopic examination in $10 \% \mathrm{KOH}$ wet mount or calcofluor white mount (fungal cell wall stain viewed under fluorescent microscope) to demonstrate septate hyphae and arthrospores (In lesions/ clinical samples, dermatophytes appear as hyphae and arthrospores/ arthroconidia), study of colony characteristics by isolating organisms on Sabouraud's dextrose agar (SDA) with chloramphenicol and cycloheximide and microscopic examination of teased mounts in lactophenol cotton blue wet mount preparations to differentiate Trichophyton, Microsporum, Epidermophyton based on morphology of the microconidia and macroconidia.

Fig 13: Direct microscopic examination in $10 \% \mathrm{KOH}$ wet mount

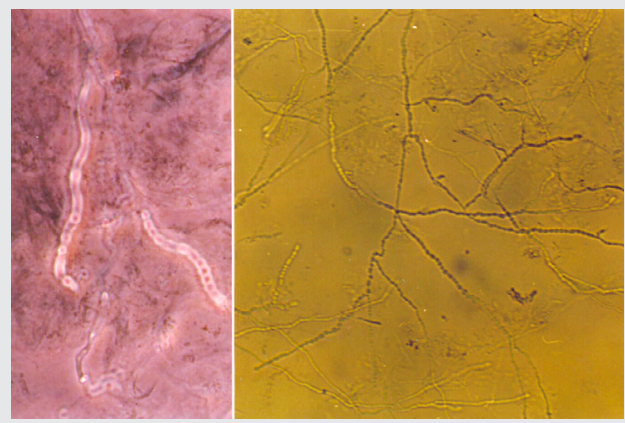

Table 2: Examination of colony characteristics

\begin{tabular}{|c|c|}
\hline 1 & \begin{tabular}{|l} 
Colony characters on the obverse \\
- $\quad$ Color (e.g., white, pearl, ivory) \\
- $\quad$ Consistency (e.g., cottony, fluffy, woolly) \\
- $\quad$ Topography (e.g., flat, folded, plicate, rugose)
\end{tabular} \\
\hline 2 & $\begin{array}{l}\text { Colony characters on the reverse } \\
\text { - Presence or absence of pigment, whether diffusing or not }\end{array}$ \\
\hline
\end{tabular}

Fig 14: Trichophyton rubrum
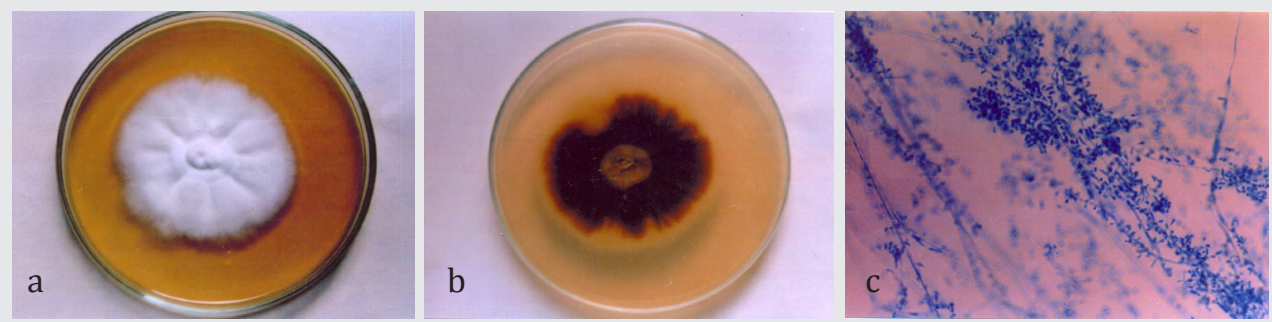

a) Obverse b) Reverse on Sabouraud's dextrose agar c) Microconidia in lactophenol cotton blue wet mounts 
Fig 15: Trichophyton mentagrophytes

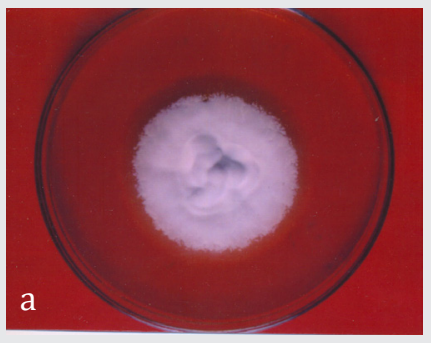

a) Obverse

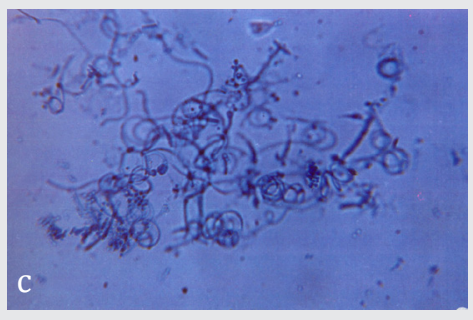

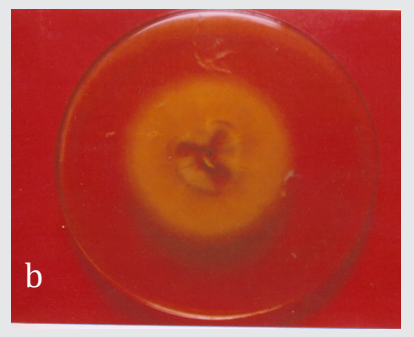

b) Reverse on Sabouraud's dextrose agar

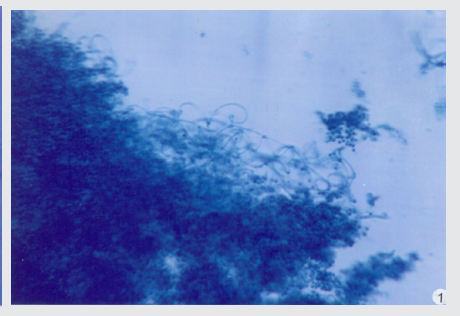

c) Spiral hyphae, rat-tail like macroconidia in lactophenol cotton blue wet mounts

Fig 16: Microsporum gypseum

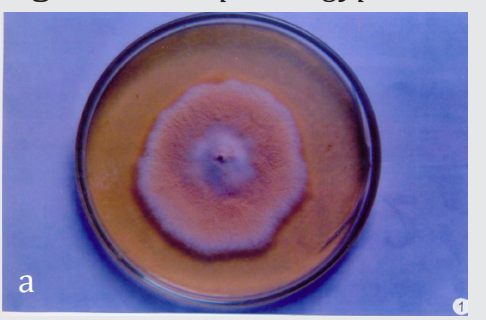

a) Fungal growth on Sabouraud's dextrose agar

\section{Fig 17: Microsporum audouinii}

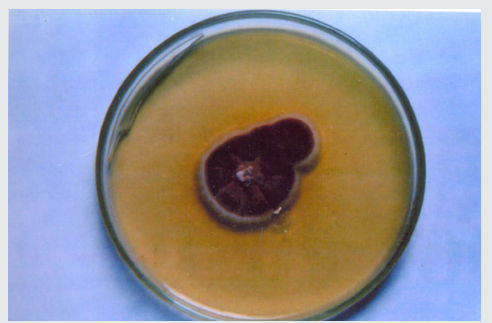

a) Fungal growth on Sabouraud's dextrose agar

Fig 18: Epidermophyton flocosum

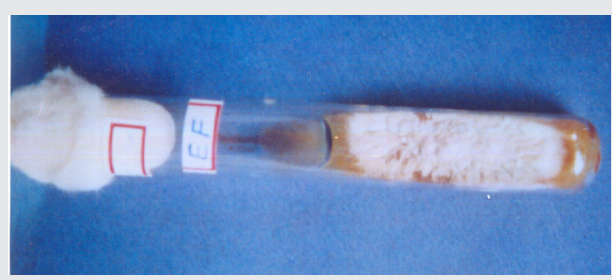

a) Fungal growth on Sabouraud's dextrose agar

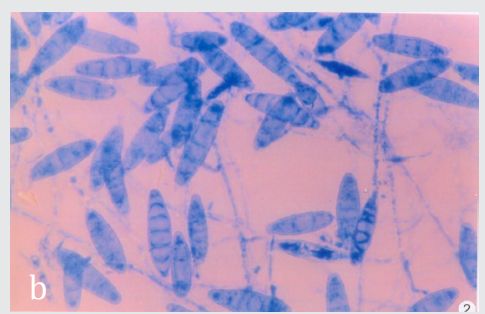

b) Large, multicellular, spindle shaped macroconidia in lactophenol cotton blue wet mounts

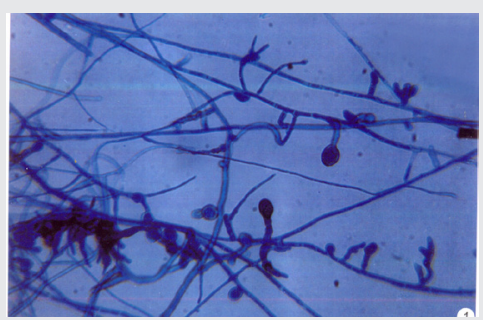

b) Chlamydospores with pectinate hyphae in lactophenol cotton blue wet mounts

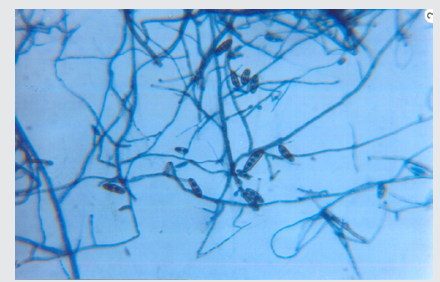

b) Macroconidia in clusters in lactophenol cotton blue wet mounts 
A dermatophyte test agar medium (DTM) is used to isolate and distinguish dermatophytes from other fungal or bacterial contaminants found in cutaneous lesions. They turn the medium red by raising the $\mathrm{pH}$ through metabolic activity while most bacteria and fungi do not (Fig 19).

Fig 19: Dermatophyte test agar

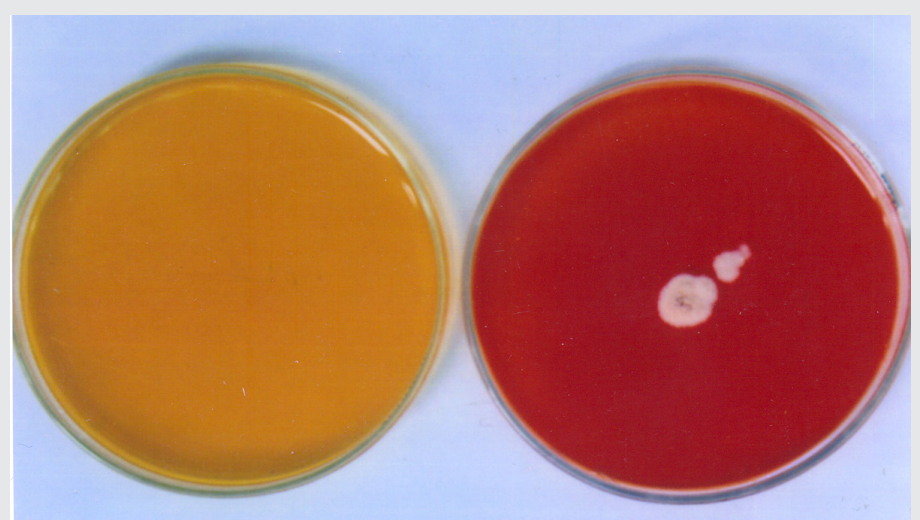

Several tests such as urease test, in vitro hair perforation tests are performed to differentiate between T. mentagrophytes and T. rubrum as well as M. canis and M. equinum. In vitro hair perforation test is taken as positive when the dermatophyte species show wedge-shaped perforations in the hair (Fig 20). It is positive in T. mentagrophytes and M. canis and negative in T. rubrum and M. equinum.

Fig 20: In vitro hair perforation test

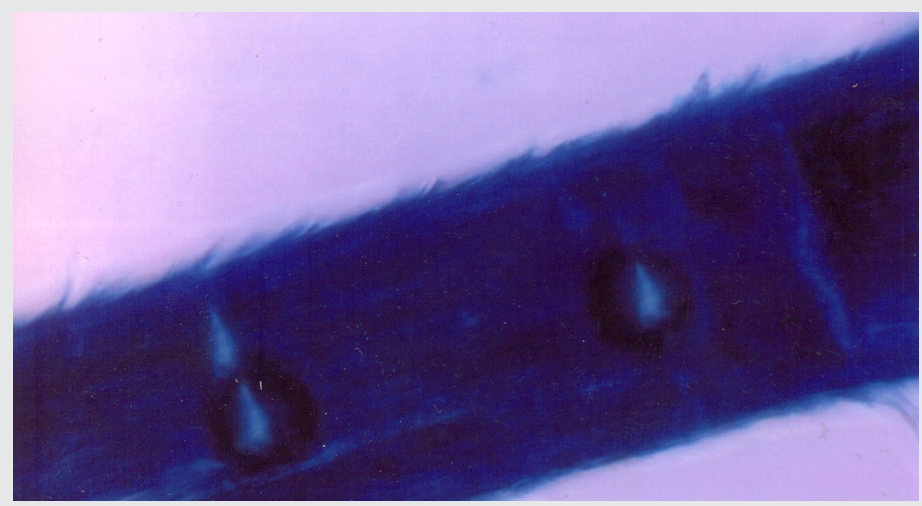

\section{Therapy}

Therapy of ring worm depends upon the site and the kind of lesion. Most infections can be treated with topical agents. Systemic therapy is used in tinea capitis, tinea unguium, wide spread invasive lesions, chronic, dry recurrent lesions or infections resistant to topical therapy.

Dermatophytes of the finger nails and toe nails in contrast to those at other body sites are particularly difficult to eradicate with drug treatment. This is the consequence of factors intrinsic at the nail i.e., hard, protective nail plate, sequestration of pathogens between the nail bed and plate and slow growth of the nail as well as relatively poor efficacy of the 
antifungal agents.

Table 2: Commonly used antifungal agents

\section{Antifungal antibiotics}

a) Polyenes

b) Others

2. Synthetic antifungal agents

a) Thiocarbamates

b) Allylamines

and benzylamines

c) Azoles

\section{Miscellaneous antifungal} agents
Amphotericin B

i. Conventional amphotericin B

ii. Lipid based formulations of amphotericin B

Nystatin

Pimaricin

Hamycin

Griseofulvin

Pradimicin

Tolnaftate

Naftifine

Terbinafine

Butenafine

i. Imidazoles

Bifonazole, Clotrimazole, Fenticonazole,

Miconazole, Oxiconazole, Butoconazole,

Econazole, Ketoconazole, Omoconazole

Sulconazole

ii. Triazoles

Fluconazole, Itraconazole,

Voriconazole, Terconazole,

Posaconazole, Ravuconazole

Flucytosine

Ciclopiroxolamine

Amorolfine

Whitfield's ointment

Potassium iodide

Selenium sulfide

Undecylenic acid

Haloprogin

Triacetin

Echinocandin

Nikkomycin

Gention violet paint

\section{Prevention and control}

Prevention and control of dermatophyte infections must take into consideration the area invaded, the etiologic agent and the source of infection. The infection could be prevented by avoiding tight fitting clothes, synthetic or wollen undergarments and socks, and closed foot wear.

Further, it is recommended to

i. Maintain good personal hygiene.

ii. Use loose clothing and absorbent undergarments and socks.

iii. Use open foot wear.

iv. Use clothing and towels which are well-laundered in hot-water, dried and ironed.

v. Dry thoroughly the intertriginous areas and apply a simple talcum powder or antifungal powder after a bath.

vi. Encourage simultaneous treatment of contacts and family members. 